\title{
ROTATING CRYSTAL CUBE AS A VARIABLE SHUTTER FOR USE WITH SYNCHROTRON RADIATION*
}

\author{
Armon McPherson, Wah-Keat Lee, and D. M. Mills \\ Experimental Facilities Division \\ Advanced Photon Source, Argonne National Laboratory \\ Argonne, IL 60439
}

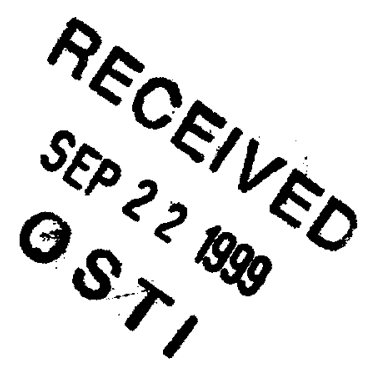

June 1998

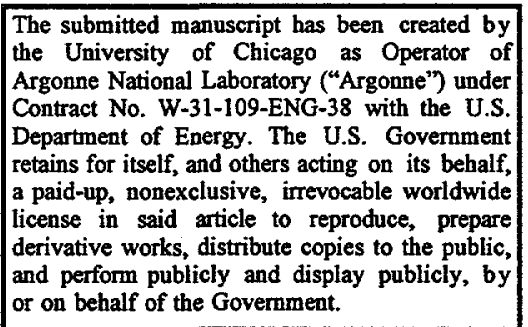

To be presented at the SPIE Conference "Time Structure of X-ray Sources and Its Applications, San Diego, CA, July 19-24, 1998, and published in the Proceedings.

*This work is supported by the U.S. Department of Energy, Basic Energy Sciences-Materials Sciences, under contract \#W-31-109-ENG-38. 


\section{DISCLAIMER}

This report was prepared as an account of work sponsored by an agency of the United States Government. Neither the United States Government nor any agency thereof, nor any of their employees, make any warranty, express or implied, or assumes any legal liability or responsibility for the accuracy, completeness, or usefulness of any information, apparatus, product, or process disclosed, or represents that its use would not infringe privately owned rights. Reference herein to any specific commercial product, process, or service by trade name, trademark, manufacturer, or otherwise does not necessarily constitute or imply its endorsement, recommendation, or favoring by the United States Government or any agency thereof. The views and opinions of authors expressed herein do not necessarily state or reflect those of the United States Government or any agency thereof. 


\section{DISCLAIMER}

Portions of this document may be illegible in electronic image products. Images are produced from the best available original document. 


\title{
Rotating crystal cube as a variable shutter for use with synchrotron radiation
}

\author{
Armon McPherson', Wah-Keat Lee, and D. M. Mills \\ Argonne National Laboratory, Experimental Facilities Division, Argonne, IL 60439
}

\begin{abstract}
A beam chopper together with the temporal structure of $x$-rays emitted by a synchrotron storage ring can be utilized to generate $x$-ray bursts of variable length and time separation. A Si cube, cut for diffraction from the (220) planes, was mounted to a low-speed motor to produce a beam chopper based upon the Darwin width of the crystal. An $x$-ray pulse, consisting of an envelope of individual pulses characterizing the loading pattern of the storage ring, was transmitted. The width of the transmitted pulse and the time between pulses was varied by varying the rotation frequency of the $\mathrm{Si}$ cube. Pulses as short as $\approx 75 \mathrm{ps}$ or as long as $\approx 4 \mu$ s were transmitted with pulse separation spanning from $4 \mathrm{~ms}$ to $167 \mathrm{~ms}$.
\end{abstract}

Keywords: time resolved, synchrotron radiation, rotating crystal, beam chopper

A synchrotron storage ring represents a unique $x$-ray light source in both the photon flux that can be delivered to a target sample and the temporal structure of the emitted $x$-rays. The temporal properties are determined by: (1) the bunch length of the stored particles in the storage ring; and (2) the specific filling pattern of the charged particles within the storage ring. The pulsed nature of the radiation can be exploited for timeresolved $x$-ray experiments. However, a given fill pattern of the storage ring may not be optimal for all types of experiments rendering the capability to modify a given time structure, determined by the fill pattern, to be highly desirable.

In the following we describe a monochromatic beam chopper capable of varying the time structure of $x$-ray pulses from a storage ring delivered to a target sample. A Si crystal cube, cut for diffraction from the (220) planes, is mounted to a low-speed motor operating asynchronously with respect to the orbital frequency of the storage ring. The beam chopper takes advantage of the small acceptance angle of the rotating crystal for diffraction, characterized by the Darwin width of the crystal, to produce an open time window to chop the $x-$ ray signal from the storage ring. The small acceptance angle for diffraction implies that even a slow rotation frequency of the crystal can achieve a short open time window. The primary advantage of this type of beam chopper over the fast shutter (typically limited to transmission times greater than $1 \mathrm{~ms}$ ) or the rotating-disk beam chopper (typically operating at high RPM to produce fast transmission times) is that a slow-speed motor may be used to produce pulses of short duration. Slow-speed motor operation is also the primary disadvantage of this type of beam chopper because of the difficulty in controlling the rotation speed accurately enough to synchronize the transmission time to a given part of the storage ring fill pattern, as discussed later.

A rotating-crystal beam chopper has been demonstrated at a synchrotron storage ring facility before, ${ }^{1}$ and proposed variations on this idea have included rotating mirrors operating at high RPM. ${ }^{2}$ In the previous case of using a rotating crystal, the temporal structure of the transmitted $x$-rays was limited by the single-bunch loading pattern employed by the storage ring at the time of the study. Here we demonstrate that a rotating-

- Further author information -

A.M. (correspondence): E-mail: armon@aps.anl.gov; Telephone: 630-252-9173; Fax: 630-252-9303 
crystal beam chopper, coupled with the multibunch loading pattern of the Advanced Photon Source at Argonne National Laboratory, can be used to produce a variable-width macro $x$-ray pulse consisting of an envelope of individual pulses. By varying the rotation frequency of the Si crystal, $x$-ray pulse envelopes can be obtained spanning the temporal range from $75 \mathrm{ps,} \mathrm{corresponding} \mathrm{to} \mathrm{a} \mathrm{pulse} \mathrm{from} \mathrm{an} \mathrm{individual} \mathrm{bunch,} \mathrm{to} \mathrm{about} 4 \mu$ s corresponding to the many bunches contained in the fill pattern of the storage ring. The spacing between these pulse envelopes was likewise varied from $4 \mathrm{~ms}$ to $167 \mathrm{~ms}$, depending upon the rotation frequency of the $\mathrm{Si}_{\mathrm{i}}$ crystal.

The experimental setup employed to test the rotating-crystal beam chopper is illustrated in Figure 1. A Si (220) double-crystal monochromator was used to select either 8-keV or 16-keV x-ray beams. The x-ray beam was directed onto a rotating $\mathrm{Si}$ crystal cube cut for diffraction from the (220) planes. This cube was mounted onto a Stanford Model SR540 Beam Chopper motor. An external precision 0-12 V voltage source was used to operate the motor through the Stanford motor controller. The diffracted $x$-ray beam signal was monitored by using a fast avalanche photodiode detector (APD), Model C30703F from EG\&G Optoelectronics Canada, and a 500MHz Tektronix Model TDS 754A Digital Scope. A HeNe laser beam was reflected off a face of the rotating $S_{i}$ cube and detected by a photodiode to monitor the approximate rotation frequency of the cube.

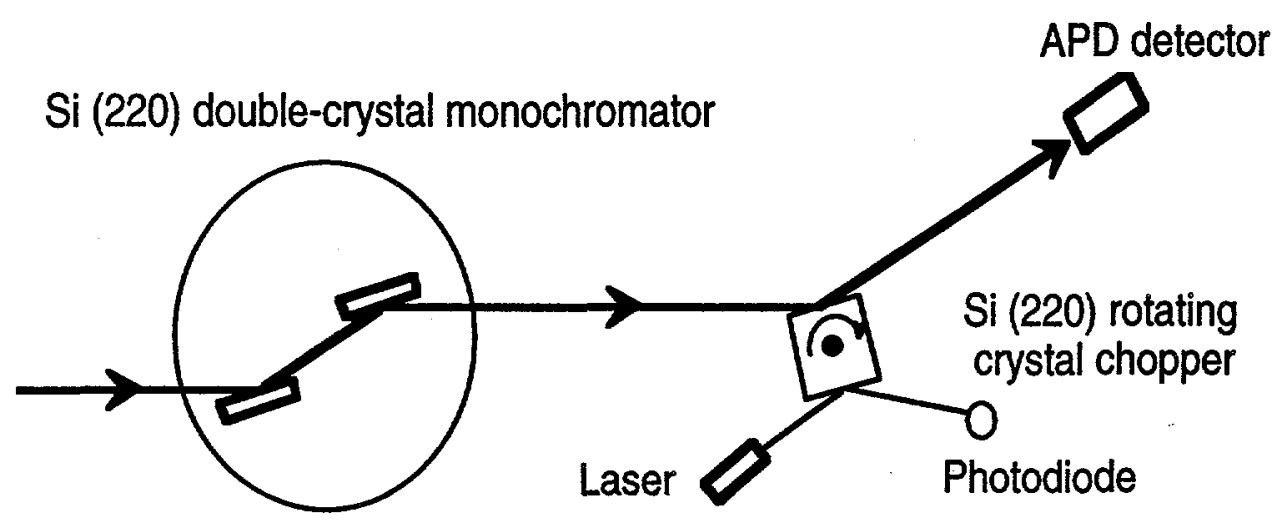

Figure. 1. Experimental setup for testing the rotating Si crystal cube beam chopper

The storage ring of the Advanced Photon Source has 1296 possible "buckets" in which particles may be stored. Presently a total of approximately $100 \mathrm{~mA}$ of current may be stored within these buckets. The width (FWHM)

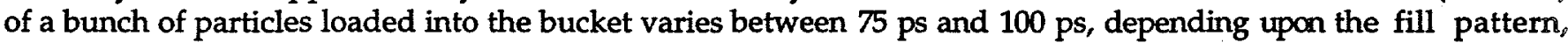
with a between bunch spacing of about $2.84 \mathrm{~ns}$, as illustrated in Figure 2. Currently the storage ring is loaded with a sextet (six adjacent buckets), a gap of about $150 \mathrm{~ns}-200 \mathrm{~ns}$, and a series of single-loaded buckets or triplets (three adjacent buckets) spaced every 100 to $150 \mathrm{~ns}$ to cover approximately $2 / 3$ of the ring space, or about $2.6 \mu \mathrm{s}$ of the $3.68 \mu \mathrm{s}$ revolution time of the storage ring. The photon flux per triplet through a $2 \mathrm{~mm}$ by $2 \mathrm{~mm}$ slit located 30 meters from the source is approximately $10^{3}-10^{4}$ photons from a bending magnet source and is $10^{7}-10^{8}$ photons from the Advanced Photon Source undulator A source. Typically 10\% of the current stored in the storage ring is contained in the sextet with the remainder of the current equally divided between the remaining buckets. Hence the photon flux from the sextet is about 3 times larger than that from a singlet or triplet.

The opening window time of the rotating-crystal beam chopper is proportional to the rocking curve width of the crystal and the rotation frequency of the crystal cube. Table 1 illustrates typical open window times for the experimental setup illustrated in Figure 1. The rocking curve widths are FWHM values. By varying the rotation frequency of the $\mathrm{Si}$ crystal, the transmitted pulse of $\mathrm{x}$-rays may be composed of individual buckets of the storage ring fill pattern or of any number of buckets up to the entire fill pattern resulting in pulses from 75-ps to several microsecond duration. The spacing between transmitted pulses decreases with increased rotation 
frequency of the crystal. In this study the spacing between pulses could be varied between and 167 ms 235 the Stanford slow-speed motor.

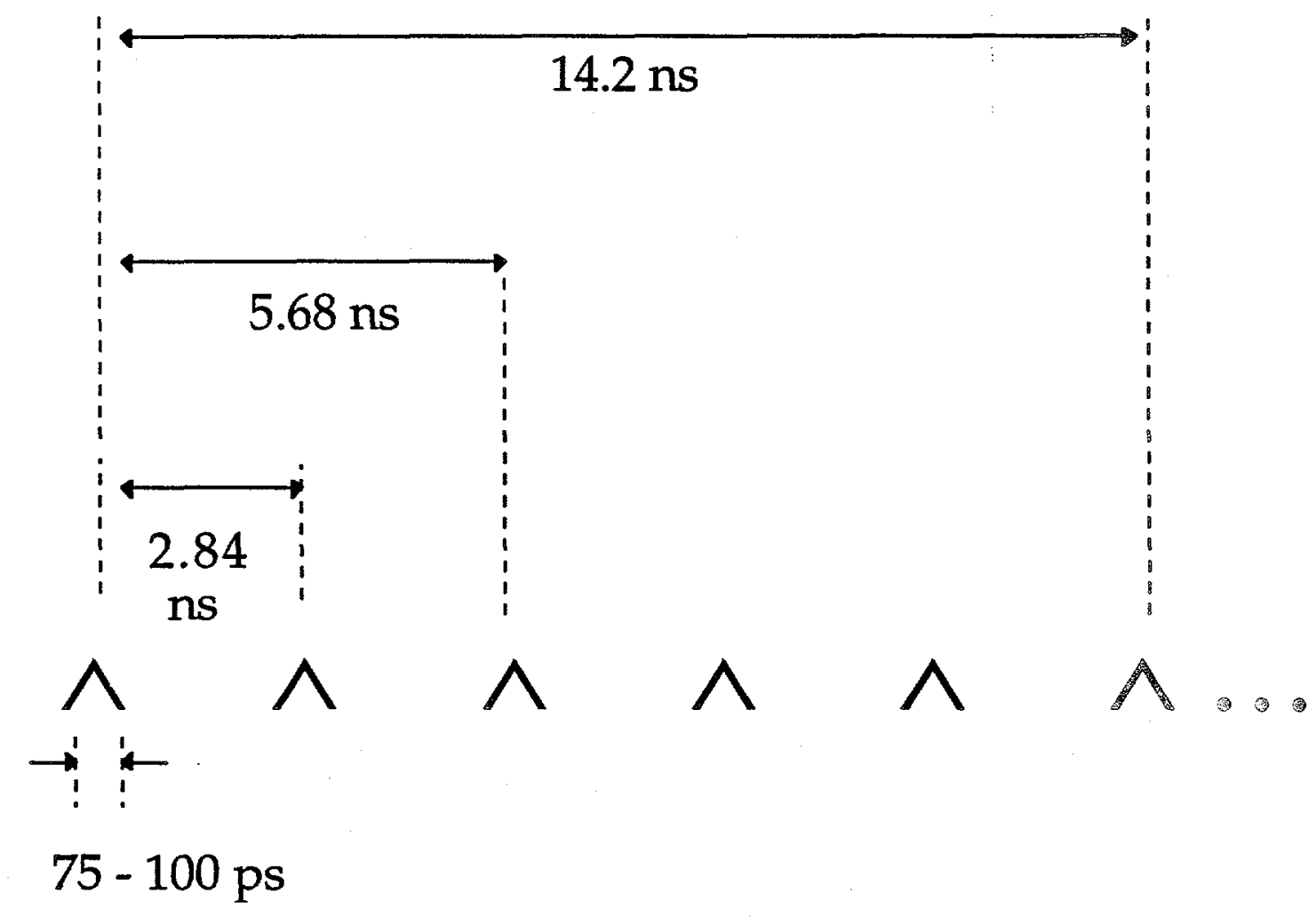

Figure 2. Symbolic representation of the bucket time structure of the storage ring at the APS for singlet, triplet and sextet loading

Table 1: The Open Window Time and Spacing Between Pulses as a Function of the Crystal Cube Rotation Frequency and the Crystal Rocking Curve Width

\begin{tabular}{ccccc}
\hline $\begin{array}{c}\text { X-Ray Energy } \\
(\mathrm{keV})\end{array}$ & $\begin{array}{c}\text { Rocking Curve } \\
\text { Width } \\
\text { (Arc sec) }\end{array}$ & $\begin{array}{c}\text { Rotation } \\
\text { Frequency } \\
(\mathrm{Hz})\end{array}$ & $\begin{array}{c}\text { Open Time } \\
\text { Window } \\
(\mathrm{ns})\end{array}$ & $\begin{array}{c}\text { Spacing } \\
\text { Between Pulses } \\
\text { (ms) }\end{array}$ \\
\hline 8 & 7.41 & 1.5 & 3800 & 167 \\
& & 8.25 & 690 & 30 \\
& 18.75 & 305 & 13 \\
16 & 62.5 & 91 & 4 \\
& 3.6 & 1.5 & 1850 & 167 \\
& & 8.25 & 337 & 30 \\
& 18.75 & 148 & 13 \\
\hline
\end{tabular}


A transmitted $x$-ray pulse occurs for each of the four faces of the rotating $\mathrm{Si}$ crystal cube. In Figure 3 are oscilloscope screen images that illustrate the temporal structure and the spacing between pulses transmitted by the beam chopper when the Si crystal is rotating at about $1.5 \mathrm{~Hz}$. In all cases the amplitude of the recorded signal should be regarded as relative or symbolic since no effort was made to calibrate the amplitude of the response of the APD to beam current in the particle bunch or to correlate the $x$-ray pulse arrival time at the $S$ i crystal to the rocking curve width of the crystal. The typical response time of the APD is less than $5 \mathrm{~ns}$, ensuring a sufficiently fast readout time to record a signal at the fastest open time window of the rotating $S_{i}$ crystal. In Figure 3(a) the Si crystal rotation of about $1.5 \mathrm{~Hz}$ results in a detected signal frequency of about $6 \mathrm{~Hz}$. The bottom trace is the HeNe laser signal used to monitor the crystal rotation frequency. (The bottom trace has been suppressed in Figures 3(b) through 3(d) for clarity.) The top trace is the signal from the 8-keV x-ray beam detected by the APD detector. Each $x$-ray pulse is about $4 \mu$ s long with a between pulse spacing of about $165 \mathrm{~ms}$.

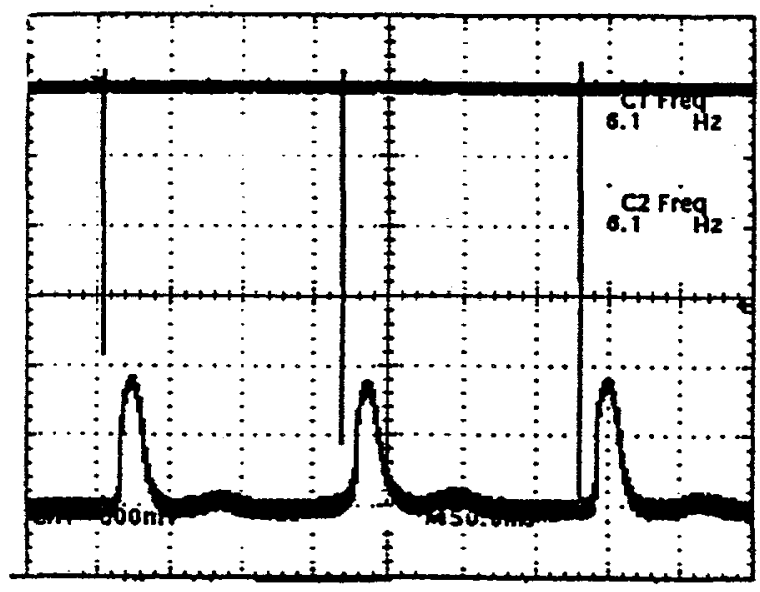

a.

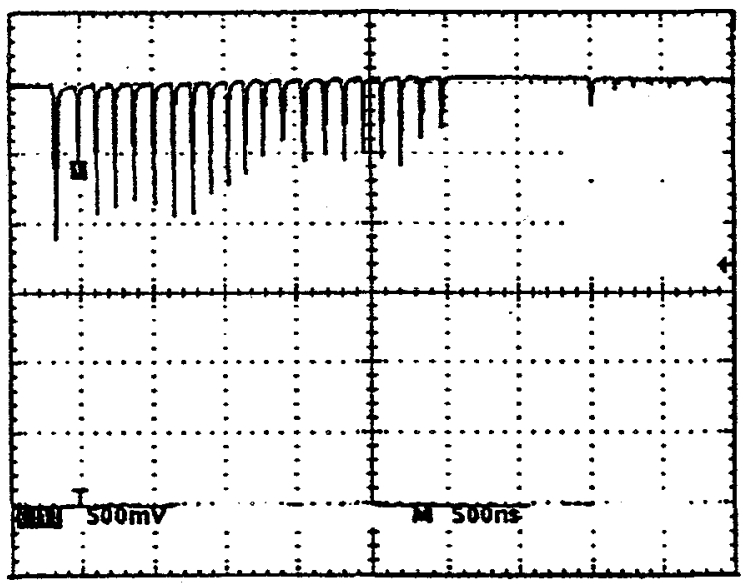

C

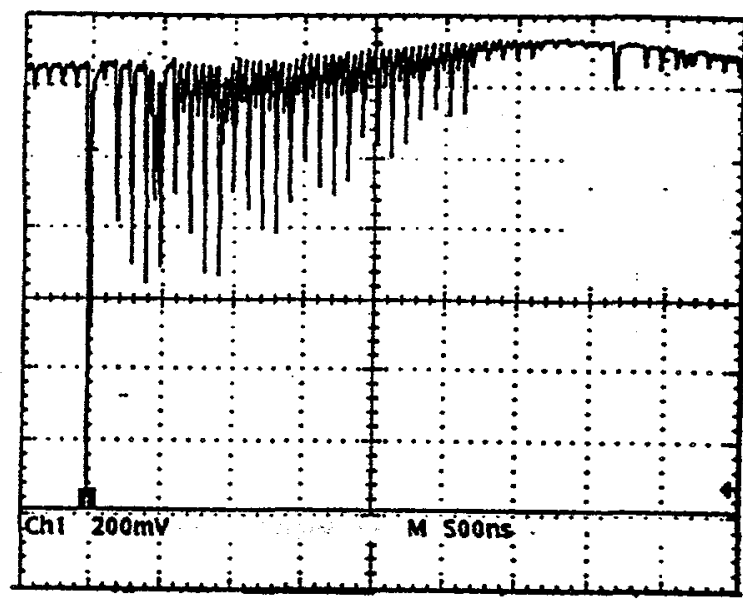

b

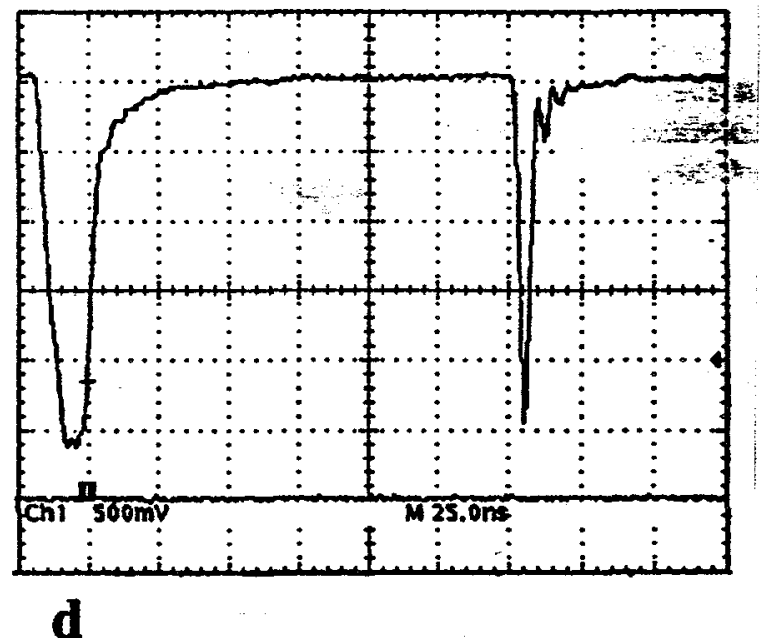

Figure 3. The time structure of the pulses transmitted by the beam chopper, when it is rotating at about $1.5 \mathrm{~Hz}$, are illustrated in these oscilloscope screen images. In (a) each pulse in the top trace represents the entire loading pattern of the storage ring followed by a dead time of about $165 \mathrm{~ms}$. In (b) and (c) the time structure of each pulse represented by the signal in (a) is illustrated for two different loading patterns of the storage ring: in (b) a sextet followed by 25 triplets and in (c) a sextet followed by 20 singlets. In (d) the time scale of the oscilloscope is expanded to illustrate the width of the sextet pulse and the following first singlet. 
Each of the pulses in Figure 3(a) is really an envelope of several pulses as shown in Figure 3(b) and Figure 3(c), where the time-scale resolution is increased. (The noise beginning about $500 \mathrm{~ns}$ after the trigger pulse in (b) is due to reflection problems resulting from impedance mismatch in the detector circuit. This problem was later corrected as illustrated by the clean pulses in (c).) The trigger pulse in each example is usually the sextet because it serves as an easily identified reference pulse. In Figure 3(b) the sextet is followed by 25 triplets covering a total time of about $2.65 \mu \mathrm{s}$. Note the beginning of a second set of pulses $3.68 \mu \mathrm{s}$ after the trigger pulse sextet, which represents transmission by the tail of the rocking curve width of the rotating crystal. Figure 3(c) illustrates a loading pattern of 1 sextet followed by 20 singlets. Note that the trigger pulse is the first singlet and that a second set of pulses begins about $3.68 \mu \mathrm{s}$ after the sextet. Increasing the time scale further, as in Figure 3(d), illustrates the characteristics of the sextet signal used to identify it, namely its increased width of about $17 \mathrm{~ns}$, and the known spacing to the next pulse within the envelope, about $165 \mathrm{~ns}$.

As the rotation frequency of the $\mathrm{Si}$ crystal is increased, the beam chopper transmission window time decreases. In Figure 4, the rotation frequency of the cube is about $62-63 \mathrm{~Hz}$ and the storage ring loading pattern is the same as illustrated in Figure 3(c) and 3(d). Each pulse in the top trace of Figure 4(a) now represents either the sextet or a singlet. The amplitude of the transmitted pulse varies because of asynchronous timing between the orbital frequency of the storage ring and the rocking curve width of the Si crystal. Note that some pulses were missed because the timing of the rotation of the Si crystal was completely out of phase with respect to the arrival of an x-ray pulse. Figure 4(b) illustrates that each pulse in Figure 4(a) represents either the sextet or a singlet because of the short open time of the transmission window, approximately $90 \mathrm{~ns}$. The first singlet following the sextet, as illustrated in Figure 3(d), is missing here because the transmission window of the beam chopper closed before arrival of the first singlet.

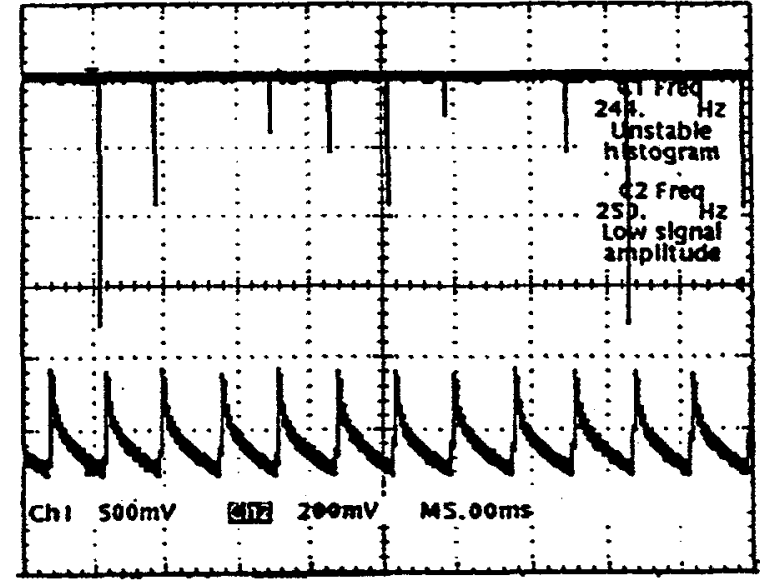

8

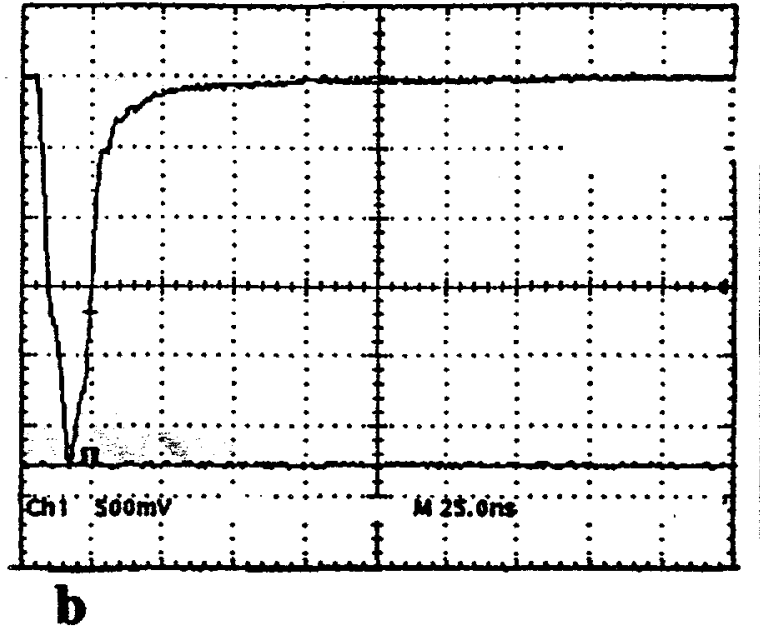

Figure 4. As the rotation frequency of the $\mathrm{Si}$ cube is increased to about $63 \mathrm{~Hz}$, the transmission window time decreases to about 90 ns. Asynchronous timing results in the variable $x$-ray pulse amplitude observed in (a). Each x-ray pulse in (a) is either the sextet or a singlet because the transmission window time is too short to transmit two adjacent pulses, as illustrated by the lone sextet in (b).

Figure 4(a) illustrates both the strength and the weakness of operating the beam chopper motor asynchronously with respect to the orbital frequency of the storage ring. On the plus side, an X-ray pulse can be transmitted with a high duty cycle (number of pluses transmitted vs. the number of possible pulses). On the negative side, the amplitude of the transmitted pulses will vary greatly from pulse to pulse depending upon the correlation between the arrival time of the $x$-ray pulse from the storage ring and the rocking curve width of the rotating crystal. For experiments that need short $x$-ray pulses, but not necessarily a constant pulse amplitude, 
asynchronous operation is an easy setup to achieve. Electronic binning of data based upon the $x$-ray pulse amplitude could be added to further refine the capabilities of the experimental setup. By far the best improvement to the operation of this beam chopper would entail running the chopper motor synchronously with respect to the storage ring orbital frequency so that one could lock onto a given singlet, or triplet, or onto the sextet to achieve a constant $x$-ray pulse amplitude. However, synchronous operation of the motor would require both high-speed electronic and mechanical precision lasting over several hours of operation, a difficult task for slow-speed motor operation.

The present rotating Si crystal beam chopper, operating asynchronously with respect to the orbital frequency of the storage ring, can utilize the temporal structure of emitted $x$-rays from the Advanced Photon Source storage ring to generate $x$-ray bursts of variable length and time separation for a variety of time-resolved experimental studies. A near term goal of this project is to demonstrate that a slow-speed rotating Si crystal beam chopper operating synchronously with respect to the orbital frequency of the Advanced Photon Source can lock onto the sextet and produce $x$-ray pulses of a constant $( \pm 10 \%)$ amplitude.

\section{ACKNOWLEDGEMENTS}

Use of the Advanced Photon Source was supported by the U. S. Department of Energy, Basic Energy Sciences, Office of Energy Research, under contract no. W-31-109-Eng-38.

\section{REFERENCES}

1. Eberhard G. Bayer, Peter Kizler and Jochen R. Schneider, "Rotating crystal pulse selector for the separation of single synchrotron radiation flashes in the X-ray regime," Nuclear Instruments \& Methods in Physical Research A313, pp. 546-548, 1992

2. J. R. Norris, M. K. Bowman, L. Chen, J. Tang, M. C. Thurnauer, G. S. Knapp and P. A. Montano, "A shutter design for time domain studies using synchrotron radiation at the Advanced Photon Source, Rev. Sci. Instrum. 63, p. 1172, 1992. 